Dieser Käsestoff der Cewebe ist in dem Blute fertig gebildet. Er braucht also durch die Wand der Haargefasse nur unverändert hindurch zu schwitzen, um in der mittleren Schlagaderwand, in dem Zellgewebe und im Nackenbande die Rolle eines Gewebebildners $2 u$ übernehmen.

\title{
XLVII.
}

\section{Ueber Pseudo-Stearoptene, welche auf der Aussenseite der Pflanzen rorkommen.}

\author{
Von \\ Moteach.
}

(Ber. d. Berl. Akad. Decbr. 1851.)

Pseudo-Stearoptene sind die durch Wärmeentziehung verdichteten Theile nüchtiger Oele und Harze, die in vierseitigen Prismen und Nadeln krystallisirbar, ziemlich hart, schwerer als Wasser, bei $50^{\circ}$ schmelzbar, bei abgehaltener Luf unverändert sublimirbar, schwach gewürzhaft-riechend und schmeckend, in warmem Wasser, Alkohol, Aether, Oelen, Essigsäure und Alkalien Joslich sind. Sie stehen zwischen den flüchtigen Oelen und Harzen in der Mitte und unterscheiden sich von den flüchtigen Oelen mit einem einfachen Kohlenwasserstoffradicale durch ihren Sauerstoffgehalt und von den wabren Stearoptenen durch ihre Löslichkeit in einer verhältnissmåssig geringen Menge heissen Wassers.

Es gehören hierher: der Alyxia-Kampher aus Alyxia aromatica (Reinw.) der Geraninm-Kampher aus Pelargonium odoratissimium (Aiton), das Coumarin (Tonka-Kampher) aus Melilotus officinalis (L.), der Ruchgras-Kampher aus Anthoxanthum odoratum (L.) und der Aurikel- oder Primel-Kampher aus Primula Auricula (L.) und mebreren anderen Arten dieser Gattung.

Bisher war die Anwesenheit der eben namhan gemachten Pseudo-Stearoptene nur innerbalb der betreffenden Pllanzen nachgewiesen, dass sie auch auf der Aussenfläche der Pfanzen angetroffen werden, war unbekannt.

Die Secretionen einer anscheinend mehlartigen, trocknen Substanz von weisser ader gelber Farbe, wie sie auf der Aussen- 
seite der Aurikel und diesen verwandte Arten der Gattung Primula und auf der unteren Fläche der Wedel von Ceropteris (Link), die von mir aber nur zur Bezeichnung einer Untergattung von Gymnogramme (Desvx.) benutzt worden ist, so wie aller übrigen Farren, die einen ähnlichen, mehlartigen Ueberzug auf der Rückseite der Wedel zeigen, vorkommen, bielt man allgemein fïr wachsartig.

Der Zufall belehrte mich, dass die hier vorkommende Secretion kein Wachs, wie es bei Stillingia sebifera (Nart.), Rhus succedanea (L.), den Myrica-Arten und bei Ceroxylon Andicola (Humb.) und C. Klopstockia (Mart.) bestimmt nachgewiesen ist, sondern ein Pseudo-Stearopten sei.

Vor bereits 4 Jahren, als ich eben mit einer Revision der Gattung Gymnogramme beschäftigt war, wollte mir es nicht glücken, von den zu der Untergattung Ceropteris gehörenden Arten, wegen des den Sporangien, die äusserst zicrlich und für die Artenbestimmungen charakteristisch sind, anhangenden, pulverigen Ueberzugs ein reines und klares Bild zu erhalten. Es kam mir jetzt darauf an, diesen pulverigen Veberzug durch ein Lüsungsmittel $z u$ beseitigen, was durch einen Zusatz von Alkohol geschab.

Während dem Verdamplen des Alkohols bemerkte ich ein Anschiessen von Nadelkrystallen, die kürzer oder länger, einzeln oder von irgend einem Punkte ansgehend, einen Bündel bildeten, der sich strahlenformig ausbreitete. Die Krystalle selbst waren ohne jede Färbung and bildeten vierseitige Prismen mit schrägen Spitzen.

Eine grössere Menge, die ich mir von dieser Substanz verschaffte, verhielt sich wie die oben angeführten Kanpherarten oder Pseudo-Stearoptene. Die gewonnenen Krystalle hatten einen eigenthümlichen, gewürzhaften Geruch und Geschmack. Die aus dem mehlartigen Ueberzige der Aurikeln erhaltener Krystalle zeigten einen schnittlauch-fenchelartigen Geruch und Geschmack.

Die Ausscheidung dieser trocknen, meblartigen Masse auf der Epidermis der Aurikeln, wie auf den Wedeln der Farren geschieht ohne Drüsen. 